\title{
Peuplement zooplanctonique du plateau continental ivoirien: diversité, abondance et biomasse
}

\author{
N'guessan Maryse AKA ${ }^{1 *}$, Raphael N'Doua ETILE ${ }^{2}$, Tapé JOANY ${ }^{1}$ et N'DA Konan ${ }^{3}$ \\ ${ }^{I}$ Centre de Recherches Océanologiques (CRO), BP V18, Abidjan, Côte d'Ivoire. \\ ${ }^{2}$ Université Félix Houphouët-Boigny de Cocody-Abidjan, UFR Biosciences, \\ Laboratoire d'Hydrobiologie, 22 BP 582 Abidjan 22, Côte d'Ivoire. \\ ${ }^{3}$ Université Nangui Abrogoua, Laboratoire de Biologie et Cytologie Animale, \\ 02 BP 801 Abidjan 02, Côte d'Ivoire. \\ "Auteur correspondant, E-mail: akanguessanmaryse@yahoo.fr
}

\section{RESUME}

La biomasse zooplanctonique représente la quantité de matière organique disponible et potentiellement transférable aux organismes du niveau trophique supérieur. La présente étude avait pour but de faire un inventaire du peuplement zooplanctonique, et d'estimer son abondance et sa biomasse du littoral ivoirien. L'échantillonnage s'est déroulé en mars 2005 dans trois stations (Assinie, Grand-Lahou et Tabou). Au total 51 taxons ont été dénombrés, dont 28 Copépodes (58\% richesse totale), 3 Cladocères (6\%) et 20 autres organismes zooplanctoniques $(39 \%)$. Au niveau quantitatif, les copépodes constituent, en moyenne, le groupe le plus abondant du peuplement, avec en moyenne $67 \%$ de l'abondance et $82 \%$ de la biomasse du zooplancton total. Les taxons dominants de copépodes en terme d'abondance sont: Paracalanus parvus $(22,18 \%$; densité moyenne : 1623 ind. $\left.\mathrm{m}^{-3}\right)$, Temora stylifera $\left(13,25 \%\right.$; 969 ind. $\left.\mathrm{m}^{-3}\right)$, Oithona plumifera $\left(11,74 \% ; 859\right.$ ind. $\left.\mathrm{m}^{-3}\right)$, Oncaea spp. $\left(10,39 \% ; 760\right.$ ind. $\left.\mathrm{m}^{-3}\right)$ et Corycaeus spp. En termes de biomasse, le groupe des Copépodes est

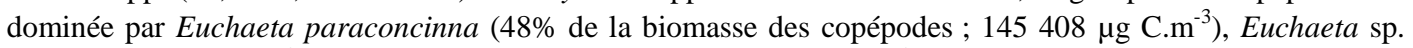
(30\%, $\left.91645 \mu \mathrm{g} \mathrm{C.m}{ }^{-3}\right)$ et Eucalanus attenuatus $\left(15 \%, 46101 \mu \mathrm{g} \mathrm{C} . \mathrm{m}^{-3}\right)$.

(c) 2018 International Formulae Group. All rights reserved.

Mots clés: Zooplancton, diversité, abondance, biomasse, plateau continental ivoirien.

\section{Ivorian Continental shelf zooplankton community:diversity, abundance and biomass}

\begin{abstract}
The zooplankton biomass represents the quantity of organic matter available and potentially transferrable to top trophic organisms. The aim of this study was to make an inventory of the zooplankton population and to estimate its abundance and biomass in the continental shelf of Côte d'Ivoire. The sampling was carried out in Assinie, Grand-Lahou, and Tabou in March 2005. A total of 51 taxa were counted, including 28 Copepods (58\%), 3 Cladocerans (6\%) and 20 other zooplankton organisms (39\%). At the quantitative level, copepods were on average the most abundant group with an average of $67 \%$ in abundance and $82 \%$ in total zooplankton biomass. The dominant taxa of copepods in terms of abundance were: Paracalanus parvus (22.18\%, mean density: 1.623 ind. $\left.\mathrm{m}^{-3}\right)$, Temora stylifera $\left(13.25 \%, 969\right.$ ind. $\left.{ }^{-3}\right)$, Oithona plumifera $(11.74 \%$,
\end{abstract}


859 ind. $\left.\mathrm{m}^{-3}\right)$, Oncaea spp. $\left(10.39 \%, 760\right.$ ind. $\left.\mathrm{m}^{-3}\right)$ and Corycaeus spp. In terms of biomass, the Copepoda group

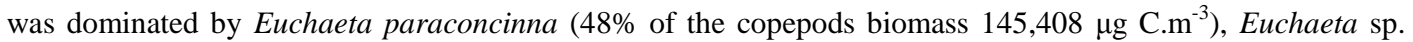
$\left(30 \%, 91,645 \mu \mathrm{g} \mathrm{C} \mathrm{m}^{-3}\right)$ and Eucalanus attenuatus $\left(15 \%, 46101 \mu \mathrm{g} \mathrm{C} \mathrm{m}^{-3}\right)$.

(C) 2018 International Formulae Group. All rights reserved.

Keywords: Zooplankton, diversity, abundance, biomass, ivorian continental shelf.

\section{INTRODUCTION}

Le zooplancton occupe une place centrale dans le réseaux trophique pélagique, assurant le transfert de matière et d'énergie issues des ressources de base (producteurs primaires phytoplanctoniques et éventuels apports allochtones) vers les niveaux trophiques supérieurs (Turner et al., 2001). D'un point de vue trophique, les communautés zooplanctoniques sont régulées d'une part par la ressource disponible, en particulier la composition de la matière en suspension (sa proportion en matière organique et inorganique), la quantité et la composition du phytoplancton, des détritus, des micro-organismes où le zooplancton trouve sa nourriture; et d'autre part, par la pression de prédation exercée par les communautés des maillons supérieurs du réseau trophique (McQueen et al., 1986) comme les poissons. Par ailleurs, en raison de sa position trophique et de son cycle de vie relativement court, le zooplancton est particulièrement sensible aux variations environnementales et répond rapidement aux changements de conditions de son milieu (Attayde et Bozelli, 1998). Ce sont donc des organismes qui sont fortement influencés, de manière directe ou indirecte, par les conditions environnementales, et sont fréquemment cités en tant qu'indicateur de pollution (Ferdous et Muktadir, 2009 ; Jakhar, 2013).

La côte d'Ivoire dispose d'une façade maritime longue de $500 \mathrm{~km}$. Au niveau de ce plateau continental, divers travaux de recherche ont été menés sur le zooplancton parmi lesquels ceux de Binet, qui datent des années 1970. Toutefois, ces travaux ont porté principalement sur : 1 - l'écologie de certains taxons [Ostracodes, Cladocères et Cirripèdes (Binet, 1975) ; Dolioles, Salpes, Appendiculaires (Binet, 1976 a); Larves de Décapodes et Lucifer (1976 b) ; Euphausiacés (Binet, 1976 c)]; 2 - sur l'estimation du biovolume et des poids secs (Binet, 1976 d) ; 3 - sur les cycles biologiques et la migration ontogénique de quelques copépodes (Binet, 1997 a) ; 4 - l'analyse globale des populations de copépodes pélagiques (Binet, 1978); et 5 sur divers autres aspects de l'études du zooplancton (Binet, 1977 b, c; 1979). Au niveau de ces différents travaux, certains taxons, notamment les plus abondants et les plus récurrents ont été identifiés parfois jusqu'au niveau spécifique. Toutefois, à ce jour, aucune liste faunistique de la composition $\mathrm{du}$ zooplancton du plateau continental de la Côte d' Ivoire n'existe. En outre, la biomasse qui représente la quantité de matière organique disponible et potentiellement transférable aux organismes du niveau trophique supérieur n'est pas encore estimée. Pour contribuer à combler ces lacunes, ce travail se propose de faire l'inventaire du peuplement zooplanctonique, d'estimer l'abondance et la biomasse du zooplancton du plateau continental ivoirien.

\section{MATERIEL ET METHODES Site d'étude}

La façade maritime de la Côte d'Ivoire occupe une portion de $566 \mathrm{~km}$ du littoral Ouest africain qui limite, au nord, le golfe de Guinée (Tastet, 1979). Elle forme la plus grande part (entre $3^{\circ}$ et $8^{\circ}$ de longitude Ouest) 
de la vaste courbe, largement ouverte vers l'atlantique sud, qui s'étend du cap des Palmes, à l'ouest, au cap des Trois-Pointes, à l'est (Figure 1). Elle peut être subdivisée en trois zones. La station d'Assinie appartient à la zone 3 qui part de Port-Bouët à la frontière du Ghana, long de 116 km, dominée par les sables. Celle de Grand-Lahou se situe dans la zone 2 qui part de Port-Bouët- Fresco, long de $190 \mathrm{~km}$, dominée par des falaises vives. Enfin la station de Tabou appartient à la zone 1 de Fresco -Tabou, long de $260 \mathrm{~km}$ dont la morphologie des plages est dominée par des roches (Tastet, 1979) (Figure 1). L'échantillonnage s'est déroulé en mars 2005 (période correspondant à la saison chaude) dans trois stations du littoral ivoirien (Assinie, Grand-Lahou et Tabou). Le zooplancton a été collecté à bord d'un navire à l'aide d'un filet cylindro-conique de $60 \mathrm{~cm}$ de diamètre d'ouverture; de 2,5 m de longueur et de 350 $\mu \mathrm{m}$ de vide de mailles. Au niveau de chaque station, trois points différents ont été échantillonnés par la réalisation d'un trait vertical sur une colonne de $100 \mathrm{~m}$. Les échantillons obtenus ont ensuite été concentrés à l'aide d'un concentreur conique, puis récupérés dans un pilulier de $500 \mathrm{ml}$ et traités selon le protocole suivant : (1) addition de 1 à $2 \mathrm{mg}$ de sucrose afin de limiter la déformation des valves des Cladocères (Haney et Hall, 1973) ; (2) - addition de formaldéhyde tamponné au borax de concentration de $5 \%$.

Les organismes zooplanctoniques ont été identifiés d'après les clés de Trégouboff et Rose (1957), Wiafe et Frid (2001), Al-Yamani et al. (2011), Conway (2012) et Conway (2015). Le comptage des taxons identifiés a été réalisé sous une loupe binoculaire de fort grossissement (Modèle Leica WILD M3C, grossissement : x160, x250 et x400) dans une cuve de Dolfuss. Les effectifs obtenus à l'issu du comptage sont ensuite exprimés en densité (nombre d'individus par $\mathrm{m}^{3}$ : ind $\mathrm{m}^{3}$ ), puis en

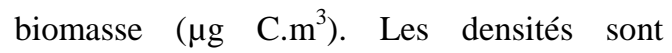
obtenues en divisant les effectifs de chaque taxon par le volume d'eau filtré $\left(\mathrm{m}^{3}\right)$ estimée en multipliant la surface d'ouverture du filet $\left(0,070685 \mathrm{~m}^{2}\right)$ par la distance de trait $(100 \mathrm{~m})$. Les biomasses sont calculées en multipliant les poids individuels de chaque taxon (exprimés en $\mu \mathrm{g} \mathrm{C}$ ) par sa densité. Les poids individuels, exprimés en microgramme de carbone, sont obtenus en multipliant les poids secs (PS) par le ratio C/PS $=0,45$ (Pagano et Saint-Jean, 1988; Pagano et Saint -Jean, 1993). Les poids secs (PS) sont estimés à partir de la relation longueur-Poids empruntée de la littérature (Tableau 1). Les tailles sont mesurées à la loupe binoculaire (objectifs x40, oculaire x10) munie d'un micromètre oculaire (précision $\pm 10 \mu \mathrm{m}, 1$ division équivaut à 25 $\mu \mathrm{m})$, sur un échantillon d'au moins 30 individus.

Les tailles considérées dans les mesures sont :

- Longueur du céphalothorax (Lc) chez les Calanides.

- Longueur totale (Lt), les soies furcales exclues chez les Cyclopides.

- Longueur totale (Lt), épine exclue chez les Cladocères.

- Longueur totale (Lt) chez les Chaetognathes, les larves de Polychaete et de Gastéropodes. Pour tous les autres taxons, les poids secs individuels sont ceux rapportés par Uye (1982), Legendre et al. (1987), Saint-Jean et Pagano (1987), Pagano et Saint - Jean (1988), Jouffre (1989) et Pagano (com. Pers.).

Les indices écologiques (indice de Shannon et indice d'équitabilité), calculés avec le logiciel PAST, ont été utilisés pour déterminer la structure et la dynamique écologique du peuplement. 


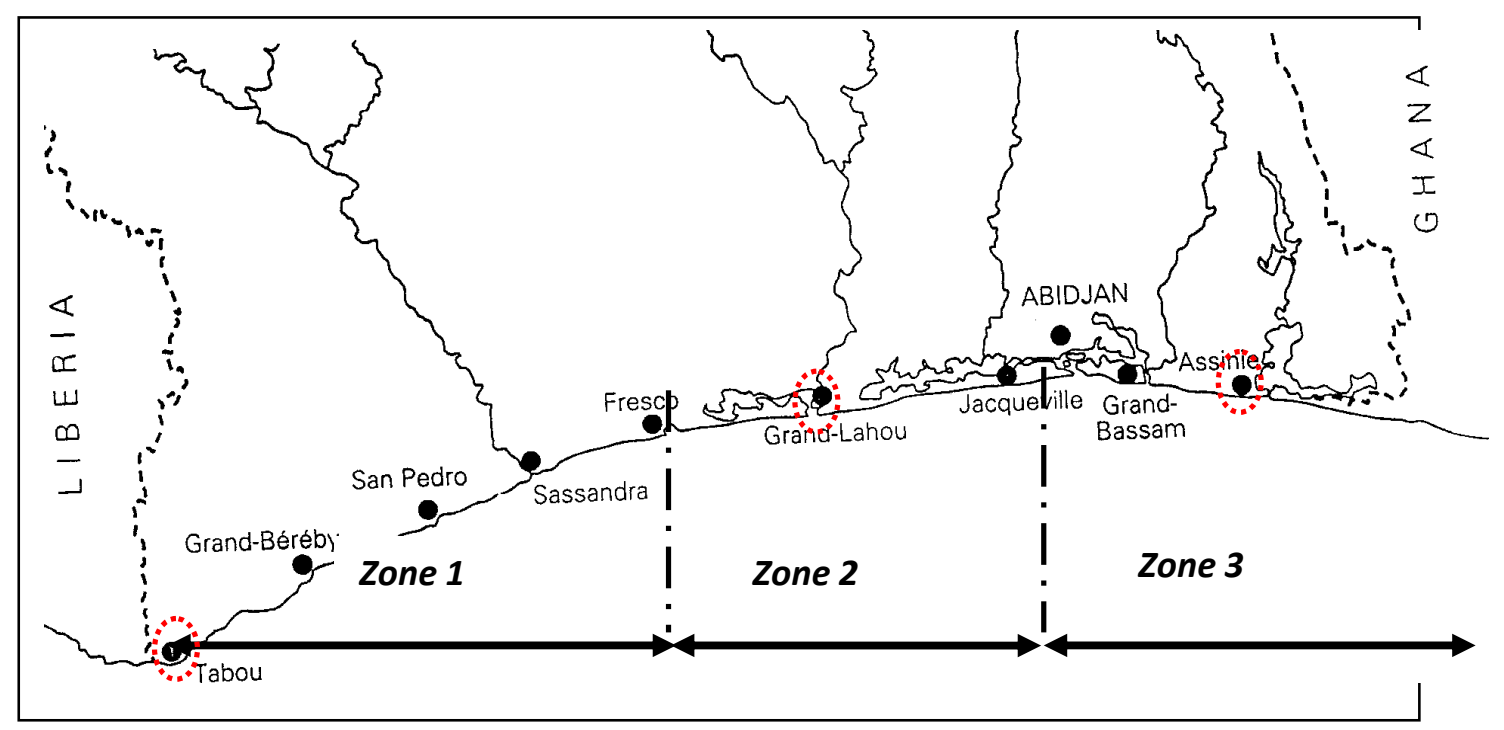

Figure 1: Zones de prélèvements des échantillons (Ecoutin et al., 1993).

Tableau 1: Formules utilisées pour le calcul des poids secs (PS) de certains taxons zooplanctoniques échantillonnés au niveau du plateau continental ivoirien.

\begin{tabular}{lccc}
\hline Groupes & Taxons & Formules (PS) & Auteurs \\
\hline & Temora spp. & PS $=24,6 \mathrm{Lc}^{2,127}$ & Nassogne (1972) \\
& Autres Calanides & PS $=17,6 \mathrm{Lc}^{2,573}$ & Nassogne (1972) \\
\hline Cyclopides & Autres Cyclopides & $\mathrm{PS}=5,7 \mathrm{~L}^{3,1}$ & Saint-Jean \& Pagano (1987) \\
\hline Cladocères & Penilia avirostris Dana 1852 & $\mathrm{PS}=15,9 \mathrm{~L}^{4,99}$ & Uye (1982) \\
\hline Autres organismes & Larve de Polychètes & $\mathrm{PS}=4 \mathrm{~L}^{1,166}$ & Nassogne (1972) \\
zooplanctoniques & Larve de Gastéropodes & $\mathrm{PS}=57,8 \mathrm{~L}^{2,362}$ & Nassogne (1972) \\
& Chaetognathes & $\mathrm{PS}=0,106 \mathrm{~L}^{3,24}$ & Uye (1982) \\
\hline
\end{tabular}

\section{RESULTATS \\ Composition, distribution et diversité du peuplement}

$\mathrm{Au}$ total, 51 taxons zooplanctoniques ont été identifiés durant cette étude (Tableau 2). Ce peuplement est composé de 28 Copépodes $(58 \%$ de la richesse taxonomique totale), 3 Cladocères marins et 20 autres formes zooplanctoniques parmi lesquelles des larves d'organismes néritiques et benthiques. Les copépodes se répartissent en 21 familles et 25 genres. Les Paracalinidae constituent la famille la plus diversifiée (6 espèces, 5 genres), suivie par les Centropagidae et les Corycaeidae, avec 2 espèces chacune. Chez les copépodes, les genres les plus diversifiés sont Paracalanus, Centropages et Macrosetella, avec chacun 2 espèces. Chez les cladocères, la communauté se compose de 3 espèces réparties en 3 genres (Evdane, Podon et Penilia) et 2 familles (Podonidae et Sididae). La famille des Podonidae, avec 2 espèces, est la plus diversifiée. Dans le groupe autres formes zooplanctoniques, la détermination n'a pas pu se faire jusqu'au niveau spécifique. Nous nous sommes très souvent arrêtés à des niveaux de détermination plus haut. Toutefois, ce groupe comprend des Euphausidae (Euphausia sp.), des Luciferidae (Lucifèr faxoni), et des 
Paneidae. Sont également compris dans ce lot des Radiolaires, des Thaliacées, des Amphipodes, des larves de poissons, des zoés de crabes, des larves de polychètes, etc. (Tableau 2).

Cette étude a révélé que la richesse taxonomique du peuplement zooplanctonique échantillonné au niveau du plateau continental ivoirien varie relativement peu (38 à 44 taxons). Vingt-neuf taxons $(57 \%$ de la diversité totale) sont communs aux trois stations d'échantillonnage (100\% d'occurrence). Pseudodiaptomus serricaudatus et Acrocalanus andersoni sont spécifiques à l'Est du littoral ivoirien d'une part (Assinie), et d"autre part Centropages violaceus et Calacalnus aculeatus sont spécifiques à la zone Ouest (Tabou) (33\% d'occurrence). Lubbockia sp. n'a été observée que dans la zone de Grand-Lahou (33\% d'occurrence). Onze taxons (22\% de la richesse taxonomique totale) ont été observés à la fois dans deux des trois stations étudiées, présentant ainsi une occurrence intermédiaire $(\approx 60 \%)$.

\section{Structure du peuplement et variation spatiale de l'abondance et de la Biomasse}

L'abondance et la biomasse moyenne $\mathrm{du}$ zooplancton total sont estimées respectivement à 10852 ind. ${ }^{-3}$ et de 36837 $\mu \mathrm{g} \mathrm{C} . \mathrm{m}^{-3}$. L'abondance et la biomasse du peuplement zooplanctonique du plateau continental ivoirien présentent une variation spatiale. L'abondance du zooplancton total augmente progressivement d'est (Assinie) à l'ouest (Tabou), passant de 6784 ind. $\mathrm{m}^{-3}$ à 13454 ind. $\mathrm{m}^{-3}$ alors que la biomasse montre une variation spatiale inverse avec une diminution progressivement d'est en ouest en passant de $119902 \mu \mathrm{g} \mathrm{C} . \mathrm{m}^{-3}$ à Assinie à $50283 \mu \mathrm{g} \mathrm{C} . \mathrm{m}^{-3}$ à Tabou (Figure $2 \mathrm{~A}$ et B).

Les Copépodes constituent, en moyenne, le groupe le plus abondant du peuplement: $67 \%$ de l'abondance et $82 \%$ de la biomasse du zooplancton total. Le groupe des copépodes est suivi du groupe autre organismes zooplanctoniques qui représente $31 \%$ de l'abondance et $18 \%$ de la biomasse totale. Au niveau des copépodes, la communauté est dominée par 5 taxons qui représentent $67,56 \%$ de l'abondance total du groupe: Paracalanus parvus $(22,18 \%$; densité moyenne: 1623 ind. $\mathrm{m}^{-3}$ ), Temora stylifera $\left(13,25 \%\right.$; 969 ind. $\left.\mathrm{m}^{-3}\right)$, Oithona plumifera $\left(11,74 \% ; 859\right.$ ind. $\left.\mathrm{m}^{-3}\right)$, Oncaea spp. $\left(10,39 \% ; 760\right.$ ind. $\left.{ }^{-3}\right)$ et Corycaeus spp. $\left(10 \% ; 732\right.$ ind. $\left.\mathrm{m}^{-3}\right)$. Paracalanus parvus et Oithona plumifera présentent les abondances les plus importantes à Tabou, avec respectivement 2992 et 1549 ind. $\mathrm{m}^{-3}$ alors que Temora stylifera et Corycaeus spp. sont observées avec les abondances les plus élevées à Grand-Lahou (respectivement 1787

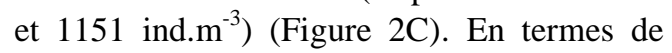
biomasse, le groupe des Copépodes est dominée par Euchaeta paraconcinna (48\% de la biomasse des copépodes ; $145408 \mu \mathrm{g} \mathrm{C.m}$ $\left.{ }^{3}\right)$, Euchaeta sp. $\left(30 \%, 91645 \mu \mathrm{g} \mathrm{C} . \mathrm{m}^{-3}\right)$ et Eucalanus attenuatus $\left(15 \%, 46101 \mu \mathrm{g} \mathrm{C} . \mathrm{m}^{-3}\right)$ qui constituent ensemble environ $97 \%$ de la biomasse totale du groupe. Les biomasses les plus élevées de Euchaeta paraconcinna ont été obtenues à Assinie $\left(270575 \mu \mathrm{g} \mathrm{C.m}{ }^{-3}\right)$ et à Tabou (145 $409 \mu \mathrm{g} \quad$ C.m ${ }^{-3}$ ) tandis que Euchaeta sp. a présenté les biomasses les plus importantes à Grand-Lahou (168 $293 \mu \mathrm{g} \mathrm{C.m}$ $\left.{ }^{3}\right)$ et à Assinie (106 $\left.641 \mu \mathrm{g} \mathrm{C} . \mathrm{m}^{-3}\right)$ (Figure 2D).

Au niveau du groupe autres organismes zooplanctoniques, les Chaetognathes, larvacés, siphonophores et les Thaliacés constituent les principaux taxons (Figure 2E). Ils représentent ensemble plus de $60 \%$ de l'abondance totale de ce groupe. Par contre, en termes de biomasse, ce groupe est dominé largement par des Euphausiacés $(84,42 \%$ de la biomasse totale du groupe ; $\left.55883 \mu \mathrm{gC} . \mathrm{m}^{-3}\right)$, suivi par des Amphipodes $(\approx 8 \% ; 5084 \mu \mathrm{g}$ C. $\mathrm{m}^{-3}$ ). Les Thaliacés et les Siphonophores ont obtenues les abondances les plus élevées à Grand-Lahou, avec respectivement 543 et 368 ind. $\mathrm{m}^{-3}$ tandis que les larvacés ont obtenu les abondances les plus élevées principalement à Tabou (1 346 ind. $\mathrm{m}^{-3}$ ) (Figure 2E). Quant aux Euphausiacés, ils ont obtenu la biomasse la plus élevée à Assinie (109359 $\left.\mu \mathrm{g} \quad \mathrm{C}^{-\mathrm{m}^{-3}}\right)$ (Figure 2F). 
Tableau 2: Liste faunistique et distribution des espèces de zooplancton récoltées dans les stations d'Assinie, Grand-Lahou et Tabou le long du littoral ivoirien.

\begin{tabular}{|c|c|c|c|c|c|}
\hline \multirow{2}{*}{ Groupes } & \multirow{2}{*}{ Familles } & \multirow{2}{*}{ Taxons } & \multicolumn{3}{|c|}{ Stations } \\
\hline & & & Assinie & Grand-Lahou & Tabou \\
\hline \multirow{3}{*}{ Cladocères } & Podonidae & Evadne tergstina claus, 1877 & + & & + \\
\hline & $"$ & Podon sp. Lilljeborg, 1853 & + & + & + \\
\hline & Sididae & Pelinia avirostris Dana 1852 & & + & + \\
\hline \multirow{19}{*}{$\begin{array}{l}\text { Copépodes } \\
\text { Calanides }\end{array}$} & Acartidae & Acartia danae Giesbrechht, 1889 & + & + & + \\
\hline & Paracalanidae & Acrocalanus andersoni Bowsman, 1958 & + & & \\
\hline & $"$ & Calocalanus aculeatus Shmeleva, 1987 & & & + \\
\hline & $"$ & Calocalanus pavo (Dana, 1852) & + & + & + \\
\hline & $"$ & Ischnocalanus plumulosus (Claus, 1863) & + & + & + \\
\hline & $"$ & Paracalanus parvus (Claus, 1863) & + & + & + \\
\hline & $"$ & Paracalanus aculeatus Giesbrechht, 1888 & + & + & + \\
\hline & Candaciidae & Candacia sp. Dana, 1846 & + & + & \\
\hline & Centropagidae & Centropages furcatus (Dana, 1849) & + & + & + \\
\hline & $"$ & Centropages violaceus (Claus, 1863) & & & + \\
\hline & Aetideidae & Chiridius poppei Giesbrechht, 1893 & + & + & + \\
\hline & Eucalanidae & Eucalanus attenuatus (Dana, 1849) & + & + & + \\
\hline & Euchaetidae & Euchaeta paraconcinna Fleminger, 1957 & + & + & + \\
\hline & $"$ & Euchaeta sp. & + & + & \\
\hline & Calanidae & Undinula vulgaris (Dana, 1849) & + & + & + \\
\hline & Lucicutiidae & Lucicutia flavicornis (Claus, 1863) & + & + & \\
\hline & Pseudodiaptomidae & Pseudodiaptomus serricaudatus (Scott T., 1894) & + & & \\
\hline & Rinhcalanidae & Rhincalanus cornutus (Dana, 1849) & + & + & + \\
\hline & Temoridae & Temora stylifera (Dana, 1849) & + & + & + \\
\hline \multirow{4}{*}{$\begin{array}{l}\text { Copépodes } \\
\text { Cyclopides }\end{array}$} & Sapphirinidae & Copilia quadrata Dana, 1849 & + & + & + \\
\hline & Corycaeidae & Corycaeus spp. Kroyer, 1848 & + & + & + \\
\hline & $"$ & Farranula gracilis (Dana, 1849) & + & + & + \\
\hline & Lubbockiidae & Lubbockia sp. Claus, 1863 & & + & \\
\hline
\end{tabular}


N'.M. AKA et al. / Int. J. Biol. Chem. Sci. 12(1): 129-140, 2018

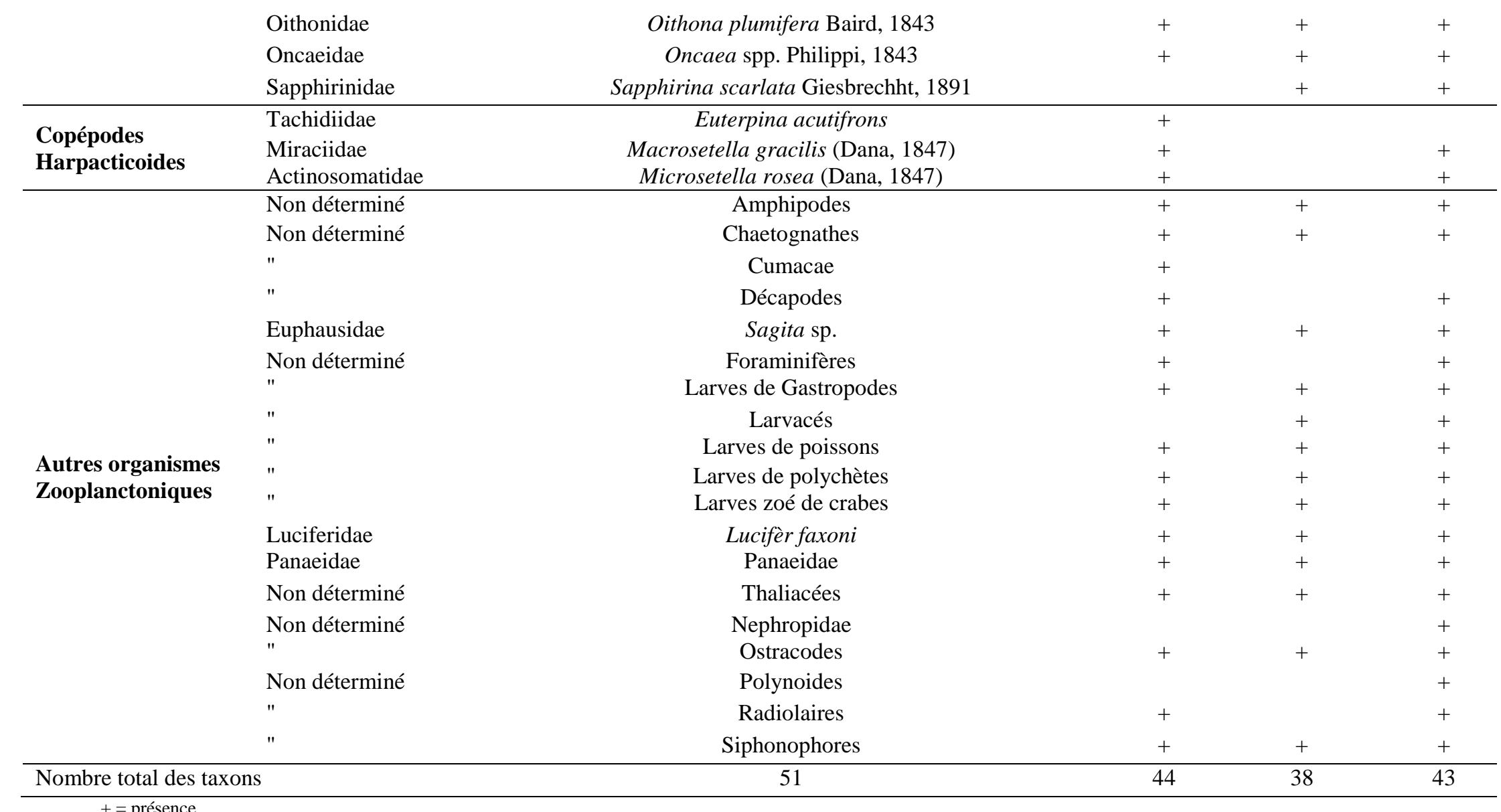



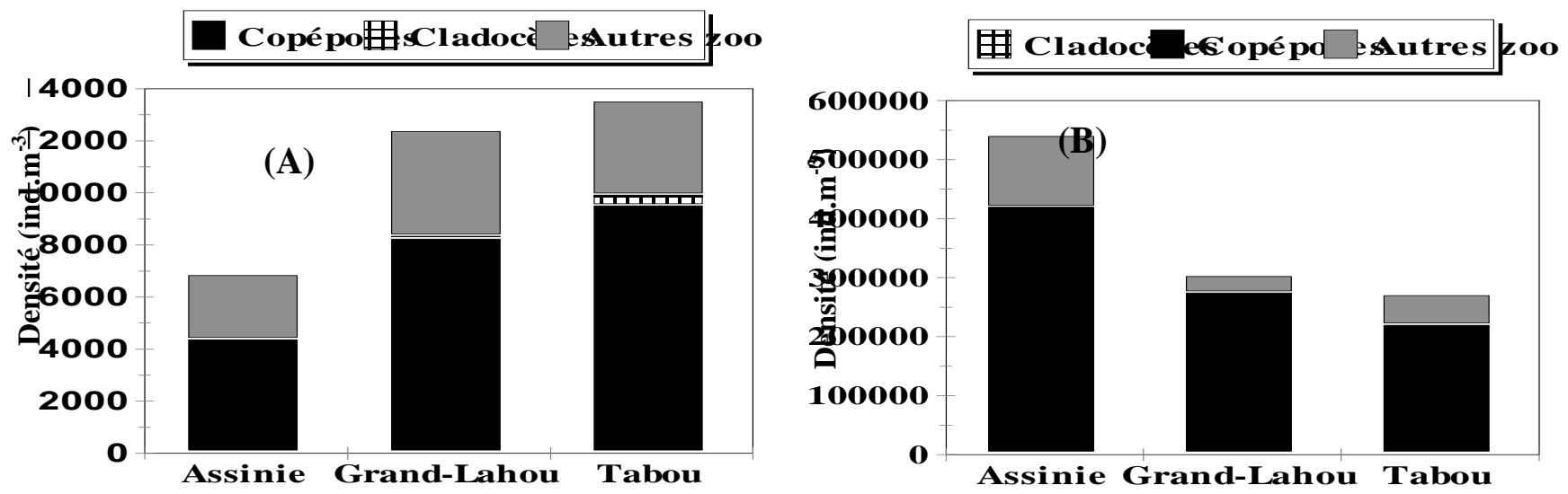

讲 Ppar $\square$ Tsty \& Cory無 Oplulll Once ACo
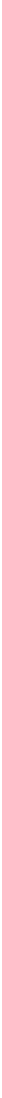

Figure 2: Variations spatiales de l'abondance (à gauche) et la biomasse (à droite) du zooplancton total (A et B), des principaux taxons des Copépodes (C et D) et du groupe autres organismes zooplanctoniques ( $E$ et $F$ ) échantillonnés au niveau du plateau continental de la Côte d'Ivoire en Mars 2005. (Ppar : Paracalanus parvus, Tsty : Temora stylifera, Oplu : Oithona plumifera, Oncea : Onceae spp., Acop : Autres copépodes, Eatt: Eucalanus attenuatus, Epara: Euchaeta paraconcinna, Euchsp.: Euchaeta sp., Chaet: Chaetognathes, Larva : Larvacées, Sipho: Siphonophores, Thal: Thaliacés, Amphi : Amphipodes, Euph : Euphausiacés, Lfax : Lucifer faxoni). 


\section{DISCUSSION}

En Côte d'Ivoire, plusieurs travaux de rechercher ont été consacrés au zooplancton du plateau continental parmi lesquels ceux de Binet des années 1970 à 1980. Malheureusement, de tous ces travaux, aucune liste faunistique des espèces n'a été dressée. Généralement, ces travaux se sont focalisés soit sur les principaux taxons (Binet, 1993), ou soit sur l'écologie des de certains taxons : Ostracodes, cladocères et Cirripèdes (Binet, 1975), Larves de décapodes et Lucifer (Binet 1976), etc. Cette étude est pour nous une contribution à l'inventaire du peuplement zooplanctonique $\mathrm{du}$ plateau continental ivoirien. Au total, 52 taxons ont été inventoriés dans les eaux du plateau continental ivoirien. Ces taxons se répartissent en 3 principaux groupes: Cladocères, Copépodes et des formes autres que celles précitées. Ce peuplement est dominé en termes qualitatif (richesse taxonomique) et quantitatif (abondance et biomasse) par les Copépodes. Ils constituent $58 \%$ de la richesse taxonomique totale (28 taxons). Ce peuplement se rapproche de celui déjà signalé par les études antérieures sur le plateau continental ivoirien (Binet, 1993) et confirme la dominance des copépodes $(68 \%$ de l'abondance total, 7317 ind. $\mathrm{m}^{-3}$ ). La richesse taxonomique de ce peuplement est cependant très largement inférieure à celle obtenue au niveau du plateau continental du Sénégal (180 espèces et plus de 50 types de larves et d'œufs) (Diouf, 1991). Elle est également inférieure à la richesse taxonomique obtenue au niveau du plateau continental du Maroc, côté océan atlantique (78 espèces, 24 familles) (Somoue et al., 2005) et à la richesse spécifique enregistrée par Araujo et Ribeiro (2008) au niveau du plateau continental du Brésil (Sergipe) (130 taxons). Cette faible richesse spécifique du peuplement zooplanctonique dans la présente étude pourrait être liée à la faiblesse de notre échantillonnage (un échantillonnage en trois sites). Elle pourrait être également en relation avec le fait que certains taxons n'ont pas pu être identifiés jusqu'au niveau spécifique (surtout dans les groupes autres organismes). La dominance des Copépodes au niveau du peuplement zooplanctonique du plateau continental Ivoirien obtenue dans cette étude confirme les résultats obtenus au niveau de la synthèse bibliographique sur le zooplancton des eaux néritiques de l'Océan Atlantique au niveau des côtes Sénégalaise (27 à 93\%) (Diouf, 1991), des côtes Atlantiques Marocaines $(73 \%)$ (Somoue et al., 2005) et Brésilienne $(\approx 50 \%$ ) (Araujo et Ribeiro, 2008).

$\mathrm{Si}$ nos travaux confirment la dominance des Copépodes dans les eaux du plateau continental ivoirien, nous observons des divergences au niveau de la structure du peuplement zooplanctonique de l'Atlantique obtenue dans des travaux antérieurs. En effet, nos travaux montrent que Paracalanus parvus constitue la principale espèce du zooplancton du plateau continental ivoirien $(24 \%, 1692$ ind. $\mathrm{m}^{-3}$ ) alors que selon Binet (1993) (Côte d'Ivoire) et Diouf (1991) (Sénégal), Calanoides carinatus constitue la principale espèce du peuplement zooplanctonique du plateau continental de ces deux pays. Au niveau de la façade Atlantique du Maroc, les espèces zooplanctonique sont Calanus helgolandicus(18 à 24\%), Acartia clausi (15\%) et Paracalanus parvus (10 à 15\%) (Somoue et al., 2015). La prédominance des taxons de la famille des Paracalanidae (avec Paracalanus quasimodo comme principale espèce) dans les eaux côtières de l'Atlantique est également relevée par les résultats des travaux d'Araujo et Ribeiro (2008) réalisés dans les eaux côtières de l'Etat de Sergipe au Brésil. Selon ces auteurs, la prédominance des taxons de la famille des Paracalanidae dans les eaux de l'Atlantique tropical pourrait être liée d'une part au fait que ces taxons sont herbivores et d'autre part au fait que les conditions favorisant la poussée phytoplanctonique peuvent être les paramètres les plus importants du modèle de la distribution des copépodes herbivores qui sont de loin les plus abondants et attirés probablement par la production $\mathrm{du}$ 
phytoplancton suscitée par plusieurs facteurs parmi lesquels les upwellings.

\section{Conclusion}

Cette étude donne pour une première fois la liste faunistique et la structure du peuplement zooplanctonique du plateau continental ivoirien. L'analyse qualitative des communautés zooplanctoniques du littoral Ivoirien en mars 2005 au niveau de trois points d'échantillonnage nous a permis d'identifier 51 taxons au total. Ce peuplement est largement dominé par les copépodes au plan qualitatif ( 28 taxons, $57 \%$ de la richesse taxonomique totale) et au plan quantitatif ( $68 \%$ de l'abondance et $82 \%$ de la biomasse totales). Les principales espèces du peuplent en terme d'abondance sont Paracalanus parvus (15\% de l'abondance totale), Témora stylifera (9\%) et Oithona plumifera (8\%). Par contre, en terme de biomasse, le peuplement est dominé par Euchaeta paraconcinna (39\% de la biomasse totale), Euchaeta sp. (24\%), Sagita sp. (15\%) et Eucalanus attenuatus $(12 \%)$.

\section{CONFLIT D'INTERETS}

Les auteurs déclarent qu'ils n'ont aucun conflit d'intérêts pour ce travail.

\section{CONTRIBUTION DES AUTEURS}

N'GMA a été l'investigateur principal. Elle a échantillonné, trié, identifié et compté les taxons à la loupe binoculaire, puis a rédigé la première version de ce présent manuscrit. RN'DE a contribué à ce travail d'abord en organisant les données pour la réalisation des graphes, puis lu et corrigé la version finale de ce manuscrit. TJ et N'DK ont participé à l'échantillonnage, à la lecture et à la correction du manuscrit.

\section{REMERCIEMENTS}

Les auteurs remercient la Direction des Productions Halieutiques (DPH) du Ministère de la Production Animale et des Ressources Halieutiques de la Côte d'Ivoire qui a favorisé la réalisation de ce travail, dans le cadre de la Campagne d'évaluation directe des ressources halieutiques marine de la Côte d'Ivoire. Ils remercient également toute l'équipe de la campagne de chalutage pour son aide et soutien durant l'échantillonnage. Enfin les éditeurs et les référés pour leur grande contribution à l'amélioration de ce présent manuscrit.

\section{REFERENCES}

Al-Yamani FY, Skryabin V, Gubanova A, Khvorov S, Prusova I. 2011. Marine Zooplankton Practical Guide for Northwestern Arabian Gulf (Vol 2). Kuwait Institute for Scientific Research (Publisher): Kuwait; 197.

Araujo HMP, Ribeiro VA. 2008. Distribution of the mesozooplankton at continental shelf off Sergipe, Brazil. Tropical Oceanography, Recife, 36(1-2): 1-13.

Attayde JL, Bozelli RL. 1998. Assessing the indicator properties of zooplankton assemblages to disturbance gradients by canonical correspondence analysis. Canadian Journal of Fisheries and Aquatic Sciences, 55(8): 1789-1797. http://dx.doi.org/10.1139/f98-033.

Binet D. 1972. Variation des biovolumes de zooplancton $\mathrm{du}$ plateau continental entre le cap des Palmes et le cap des Trois-Pointes. Document Scientifique Centre de Recherches Océanographiques Abidjan, 31(2): 6093.

Binet D. 1973. Copépodes pélagiques du plateau ivoirien. Il - Utilisation de l'arbre de longueur minimum dans le classement des récoltes. Document Scientifique Centre de Recherches Océanographiques Abidjan, 412: 1-16.

Binet D. 1975. Notes sur l'écologie de quelques taxons du zooplancton de Côted'Ivoire. 1 - Ostracodes, Cladocères et Cirripèdes. Document Scientifique Centre de Recherches Océanographiques Abidjan, 61(2): 1939.

Binet D. 1976a. Contribution à l'écologie de quelques taxons du zooplancton de Côte d'Ivoire. II-Dolioles, Salpes, 
Appendiculaires. Document Scientifique Centre de Recherches Océanographiques Abidjan, 7(1) : 45-61.

Binet D. 1976b. Contribution à l'écologie de quelques taxons du zooplancton de Côte d'Ivoire. Ill- Larves de Décapodes et Lucifer. Document Scientifique Centre de Recherches Océanographiques Abidjan, 71(1): 63-84.

Binet D. 1976c. Contribution à l'écologie de quelques taxons du zooplancton de Côte d'Ivoire. IV- Euphausiacés. Document Scientifique Centre de Recherches Océanographiques Abidjan, 7(1): 85-90.

Binet D. 1976d. Biovolumes et poids secs zooplanctoniques en relation avec le milieu pélagique au-dessus du plateau ivoirien. Cahier ORSTOM, série Océanographie, 14(4): 301-326.

Binet D. 1977a. Cycles biologiques et migrations ontogéniques chez quelques Copépodes pélagiques des eaux ivoiriennes. Cahier ORSTOM, série Océanographie, 15(12): 111-138.

Binet D. 1977b. Contribution à la connaissance du zooplancton néritique ivoirien. Ecologie descriptive et dynamique. Thèse de Doctorat d'État, Université Paris-VI, 282 p.

Binet D. 1977c. Grands traits de l'écologie des principaux taxons du zooplancton ivoirien. Cahier ORSTOM, série Océanographie, 15(12): 89-1 09.

Binet D. 1978. Analyse globale des populations de Copépodes pélagiques du plateau continental ivoirien. Cahier ORSTOM, série Océanographie, 16(1): 19-61.

Binet D. 1979. Le zooplancton du plateau continental ivoirien. Essai de synthèse écologique. Oceanologica Acta, 2(4): 397-410.

Conway DVP. 2012. Marine Zooplankton of Southern Britain (Part 1) Radiolaria, Heliozoa, Foraminifera, Ciliophora, Cnidaria, Ctenophora, Platyhelminthes, Nemertea, Rotifera, Mollusca. AWGJ (eds). Marine Biological Association of the United Kingdom, No. 25: Plymouth, United Kingdom; 138.

Conway DVP. 2015. Marine Zooplankton of southern Britain (Part 3). Ostracoda, Stomatopoda, Nebaliacea, Mysida, Amphipoda, Isopoda, Cumacea, Euphausiacea, Decapoda, Annelida, Tardigrada, Nematoda, Phoronida, Bryozoa, Entoprocta, Brachiopoda, Echinodermata, Chaetognatha, Hemichordata and Chordata AWGJ (eds). Marine Biological Association of the United Kingdom, No. 27: Plymouth, United Kingdom; 271.

Diouf PS. 1991. Le zooplancton au Sénégal, 103-116.

Ferdous Z, Muktadir AKM. 2009. A Review: Potentiality of Zooplankton as Bioindicator. American Journal of Applied Sciences, 6(10): 1815-1819.

Haney JF, Hall DJ. 1973. Sugar-coated Daphnia: a preservation technique for Cladocera. Limnol. Oceanogr., 18: 331333.

Jakhar P. 2013. Role of phytoplankton and zooplankton as health indicators of aquatic ecosystem: A review. International Journal of Innovation Research Study, 2(12): 489-500.

Jouffre D. 1989. Etude de l'organisation spatiale du zooplancton de l'étang de Thau (France) et de l'influence entre la lagune et la mer. Thèse de Doctorat, Université Montpellier II, 194.

Landa GG, Barbosa FAR, Rietzler AC, Barbosa PMM. 2007. Thermocyclops decipiens (Kiefer, 1929) (Copepoda, Cyclopoida) as indicator of water quality in the State of Minas Gerais, Brazil. Brazilian Archives of Biology and Technology, 50(4): 695-705.

Legendre M, Pagano M, Saint Jean L. 1987. Peuplements et biomasse zooplanctonique dans des étangs de pisciculture lagunaire (Layo, Côte d'Ivoire). Etude de la recolonisation après la mise en eau. Aquaculture, 67: 321-341. 
Mc Queen DG, Post JR, Mills ER. 1986. Trophic relationships in freshwater pelagic ecosystems. Canadian Journal of Fisheries and Aquatic Sciences, 43: 1571-1581.

Nassogne A. 1972. Etude préliminaire sur le rôle du zooplancton dans la constitution et le transfert de la matière organique au sein de la chaine alimentaire marine en mer ligure. Publication $N^{\circ}$ B.I.O. 187 Direction Biologie Euraton, 238 p.

Pagano M, Saint-Jean L. 1988. Importance et rôle du zooplancton dans une lagune tropicale, lagune Ebrié (Côte d'Ivoire) : peuplement, biomasse, production et bilan métabolique. Thèse, Université Aix-Marseille II, 390 P.

Saint-Jean L, Pagano M, 1987. Taille et poids individuels des principaux taxons du zooplancton lagunaire ivoirien: lagune Ebrié ; étangs de pisciculture saumâtres de layo. Revue d'Hydrobiologie Tropicale, 20(1): 13-20.

Somoue L, Elkhiati N, Ramdani M, LamHoai T, Ettahiri O, Berraho A, Dochi T. 2008. Abundance and structure of copepod communities along the Atlantic coast of southern Morocco. Acta Adriat, 46(1): 63-76.
Tastet JP. 1979. Environnements sédimentaires et structuraux quaternaires du littoral du Golfe de guinée (Côte d'Ivoire, Togo, Benin). Thèse de Doctorat d'Etat, Université de BordeauxI, 2 tomes, $181 \mathrm{p}$.

Turner JT, Ianora A, Miralto A, Laabir M, Esposito F. 2001. Decoupling of copepod grazing rates, fecundity and egg-hatching success on mixed and alternating diatom and dinoflagellate diets. Mar. Ecol. Progr. Ser., 220: 187199.

Trégouboff G, Rose M. 1957. Manuel de Planctonologie Méditerranéenne, Tome 1, Tome 2: CNRS: Paris ; 587.

Uye S. 1982. Length-Weight Relationships of Important Zooplankton from the Inland Sea of Japan. Journal of the Oceanographical Society of Japan, 38: 149-158.

Wiafe G, Frid CLJ. 2001. Marine Zooplankton of West Africa. In Marine biodiversity capacity building the West African sub-region. Darwing Initiative report 5, ref. 162/7/451. 